\title{
Recombinant Factor Vlla in Major Abdominal Surgery and Liver Transplantation
}

\author{
J. da Silva Viana
}

\begin{abstract}
The author reviewed the literature regarding recombinant activated Factor VII (rFVIIa) in major abdominal surgery and liver transplantation and concluded that, on the basis of evidence-based medicine, there is no evidence to support an extensive use of rFVIIa. Nevertheless, various case reports suggest the usefulness of rFVIIa to treat life-threatening bleeding after failure of conventional therapies. It appears that there is a consensus that rFVIIa can be used with good results as a rescue therapy in extremely severe situations. Economic cost and potential thrombosis risk remain arguments against more widespread use of rFVIIa. Doses from 5 to $120 \mathrm{~kg} / \mathrm{kg}$ in each administration have been reported without clear evidence to support a specific protocol. Efficacy of 15 to $20 \mathrm{~kg} / \mathrm{kg}$ in surgical settings has been reported, but higher doses are more frequently used. The majority of the reviewed investigators accepted the use of rFVIIa after or simultaneously with the use of aprotinin; no data refute the safety of this association.
\end{abstract}

$\mathrm{O}$ NLY ONE randomized clinical trial (RCT), including 185 patients, has evaluated the use of recombinant activated Factor VII (rFVIIa) in liver surgery. Compared with placebo there was a tendency toward fewer transfusions - the primary end point—, but it did not show statistical significance. ${ }^{1}$

\section{OTHER ABDOMINAL SURGERIES}

Extrapolating to other kinds of surgeries, an RCT including 36 patients reported a significant reduction in blood loss associated with the use of rFVIIa in retropubic prostatectomy. ${ }^{2}$ A retrospective case-controlled study compared 51 patients with 51 matched controls, observing similar results in cardiac surgery. ${ }^{3}$

If controlled studies are scarce, a relevant number of uncontrolled reports exist about the use of rFVIIa in cirrhotic patients, including those undergoing percutaneous liver biopsy, ${ }^{4,5}$ laparoscopic liver biopsy, ${ }^{6}$ endoscopic retrograde cholangiopancreatography, ${ }^{7}$ and esophageal variceal bleeding. ${ }^{8,9}$ With respect to other kinds of patients and other types of surgical interventions, the utility of rFVIIa has been advocated in cardiac surgery, ${ }^{10}$ orthopedics, intensive care, trauma, ${ }^{11}$ gynecological/obstetrical surgery, and neurosurgery.

\section{EVIDENCE ABOUT FACTOR VIla IN} LIVER TRANSPLANTATION

A preliminary study compared six patients who received rFVIIa with six matched controls. It advocated the useful- ness of the drug to reduce intraoperative $\mathrm{RBC}$ and FFP needs in liver transplantation. ${ }^{12}$ Another preliminary controlled but nonrandomized study reported the opposite results. ${ }^{13}$

Beside these studies, some case reports have been published. ${ }^{14}$ In general, no complications have been reported to be related to the use of rFVIIa, except a recent description of two thrombotic events observed with its use during four liver transplants. ${ }^{15}$ The possibility of increased thrombotic events, particularly increased vascular complications at the graft anastomosis, is a matter of concern. A study to refute this hypothesis must include a sufficient number of patients, a need that is difficult to reach in liver transplantation. For example, if the drug produces a 50\% increase in complications above the usual 5\% incidence, it will be necessary to enroll almost 1500 patients to prove this harm as statistically significant.

\section{WHEN TO USE rFVIla?}

The use of rFVIIa in surgical settings has been advocated when other measures fail to stop relevant blood loss, the socalled intractable bleeding. Some variation may be observed about this definition, but, in general, investigators defend

From the Faculty of Medicine, University of Coimbra, Coimbra, Portugal.

Address reprint requests to Prof. Joaquim Viana, Department of Anesthesiology, University Hospitals of Coimbra, Praceta Mota Pinto, 3000-075 Coimbra, Portugal. E-mail: jvviana@huc.min-saude.pt

(C) 2006 by Elsevier Inc. All rights reserved. 360 Park Avenue South, New York, NY 10010-1710 
the use of rFVIIa if bleeding persists after failure of correction with plasma, platelets, and antifibrinolytic drugs. The administration of rFVIIa after aprotinin is frequently reported; there seems to exist no harm from this combination.

In specific situations, rFVIIa may be utilized as an adjuvant to standard therapies (particularly FFP), when rapid correction of hemostasis is necessary and renal insufficiency precludes the administration of a large volume of fluids.

A final group of indications are situations where nearnormal coagulation is only transiently required. The paradigm of this situation is the insertion of sensors for intracranial pressure in patients with acute liver failure.

\section{HOW MUCH rFVIIa TO USE?}

Reports describe the use of a broad range of doses, from 5 to $120 \mu \mathrm{g} / \mathrm{kg}$ at each administration. Doses of 15 to $20 \mu \mathrm{g} / \mathrm{kg}$ seem to be useful to correct coagulation in surgical settings, although, more frequently, investigators have reported higher doses, from 40 to $90 \mu \mathrm{g} / \mathrm{kg}$. Some researchers claim advantages with the use of these high doses, but the evidence suggests that compared with $20 \mu \mathrm{g} / \mathrm{kg}, 60$ to $80 \mu \mathrm{g} / \mathrm{kg}$ increased the duration of action, ${ }^{6}$ but only produced modest benefits in efficacy.

Among cirrhotic patients, $5 \mu \mathrm{g} / \mathrm{kg}$ shows an ability to correct the prothrombin time to near-normal values. Its use has been defended for minor procedures such as percutaneous liver biopsy. ${ }^{4}$

\section{OUR GUIDELINES}

We conclude that several publications suggest the usefulness of rFVIIa to treat coagulation in surgical settings, but this has not been proven. Despite that, we consider it ethical to use rFVIIa when bleeding is a life-threatening event and when other measures, including FFP, platelet concentrates, and aprotinin, were previously unsuccessful.

We administer 15 to $20 \mu \mathrm{g} / \mathrm{kg}$ (usually $1.2 \mathrm{mg}$ to a normal adult) and repeat the dose 3 to 4 hours later, if necessary. In the choice of this dose, economics had some weight, but it was influenced by other factors: benefits claimed for high doses did not seem relevant enough to justify their use, and a short duration of action may be advantageous when there is an increased risk of thrombosis. Particularly after reperfusion in liver transplantation and in recently transplanted patients, we do not need completely normal coagulation. A certain degree of hypocoagulability, within an acceptable risk to the patient, is advantageous to reduce the incidence of any vascular complication of the graft, a life-threatening problem itself. In these conditions, rFVIIa reaches the status of a rescue therapy limited in time to periods where bleeding is considered to be a risk to the patient's life. We consider the existence of surgical conditions that predispose to thrombosis to be a relative contraindication, to be weighed against the presumed advantages.

\section{FUTURE USE OF rFVIla}

The use of rFVIIa as rescue therapy and/or its inclusion in a first-line protocol for rapid correction of coagulation at the start of liver transplant are two points that need separate further studies. Economics could be an objection to the widespread use of this drug. If the future brings evidence supporting the efficacy of rFVIIa, the doses and frequency of administration necessary to reduce RBC and FFP consumption could make a strong difference concerning wider use.

\section{ACKNOWLEDGMENTS}

We thank Dr. Emanuel Furtado for advice and valuable suggestions during the preparation of this report.

\section{REFERENCES}

1. Lodge JP, Jonas S, Oussoultzoglou E, et al: Recombinant coagulation factor VIIa in major liver resection: a randomized, placebocontrolled, double-blind clinical trial. Anesthesiology 102:269, 2005

2. Friederich PW, Henny CP, Messelink EJ, et al: Effect of recombinant activated factor VII on perioperative blood loss in patients undergoing retropubic prostatectomy: a double-blind placebo-controlled randomised trial. Lancet 361:201, 2003

3. Karkouti K, Beattie WS, Wijeysundera DN, et al: Recombinant factor VIIa for intractable blood loss after cardiac surgery: a propensity score-matched case-control analysis. Transfusion 45:26, 2005

4. Carvalho A, Leitao J, Louro E, et al: Small dose of recombinant factor VIIa (rFVIIa) to perform percutaneous liver biopsies in cirrhotic patients. Rev Esp Enferm Dig 94:280, 2002

5. Kamphuisen PW, Wiersma TG, Mulder CJ, et al: Pluggedpercutaneous liver biopsy in patients with impaired coagulation and ascites. Pathophysiol Haemost Thromb 32:190, 2002

6. Jeffers L, Chalasani N, Balart L, et al: Safety and efficacy of recombinant factor VIIa in patients with liver disease undergoing laparoscopic liver biopsy. Gastroenterology 123:118, 2002

7. Kositchaiwat C, Chuansumrit A: Experiences with recombinant factor VIIa for the prevention of bleeding in patients with chronic liver disease undergoing percutaneous liver biopsies and endoscopic retrograde cholangiopancreatography (ERCP). Thromb Haemost 86:1125, 2001

8. Ejlersen E, Melsen T, Ingerslev J, et al: Recombinant activated factor VII (rFVIIa) acutely normalizes prothrombin time in patients with cirrhosis during bleeding from oesophageal varices. Scand J Gastroenterol 36:1081, 2001

9. Romero-Castro R, Jimenez-Saenz M, Pellicer-Bautista F, et al: Recombinant-activated factor VII as hemostatic therapy in eight cases of severe hemorrhage from esophageal varices. Clin Gastroenterol Hepatol 2:78, 2004

10. Karkouti K, Beattie WS, Wijeysundera DN, et al: Recombinant factor VIIa for intractable blood loss after cardiac surgery: a propensity score-matched case-control analysis. Transfusion 45:26, 2005

11. Martinowitz U, Kenet G, Segal E, et al: Recombinant activated factor VII for adjunctive hemorrhage control in trauma. J Trauma 51:431, 2001

12. Hendriks HG, Meijer K, de Wolf JT, et al: Reduced transfusion requirements by recombinant factor VIIa in orthotopic liver transplantation: a pilot study. Transplantation 71:402, 2001

13. De Gasperi A, Baudo F, De Carlis L: Recombinant FVII in orthotopic liver transplantation (OLT): a preliminary singlecentre experience. Intensive Care Med 31:315, 2005

14. Lisman T, Leebeek FW, Meijer K, et al: Recombinant factor VIIa improves clot formation but not fibrolytic potential in patients with cirrhosis and during liver transplantation. Hepatology 35:616, 2002

15. Pavese P, Bonadona A, Beaubien J, et al: FVIIa corrects the coagulopathy of fulminant hepatic failure but may be associated with thrombosis: a report of four cases. Can J Anaesth 52:26, 2005 